This item was submitted to Loughborough's Research Repository by the author.

Items in Figshare are protected by copyright, with all rights reserved, unless otherwise indicated.

\title{
Optimising the energy efficiency and transient response of diesel engines through an electric turbocharger
}

\author{
PLEASE CITE THE PUBLISHED VERSION
}

https://ieeexplore.ieee.org/document/8815017

PUBLISHER

IEEE

VERSION

AM (Accepted Manuscript)

\section{PUBLISHER STATEMENT}

(c) 2019 IEEE. Personal use of this material is permitted. Permission from IEEE must be obtained for all other uses, in any current or future media, including reprinting/republishing this material for advertising or promotional purposes, creating new collective works, for resale or redistribution to servers or lists, or reuse of any copyrighted component of this work in other works.

\section{LICENCE}

\section{All Rights Reserved}

\section{REPOSITORY RECORD}

Zhao, Dezong, Richard Stobart, and Byron Mason. 2019. "Optimising the Energy Efficiency and Transient Response of Diesel Engines Through an Electric Turbocharger”. figshare. https://hdl.handle.net/2134/37343. 


\title{
Optimising the Energy Efficiency and Transient Response of Diesel Engines through an Electric Turbocharger
}

\author{
Dezong Zhao, Senior Member, IEEE, Richard Stobart, Member, IEEE and Byron Mason
}

\begin{abstract}
The electric turbocharger provides great potential for vehicle fuel efficiency improvement, exhaust emissions reduction and transient response acceleration. It makes the engine runs as a hybrid system so critical challenges are raised in energy management and control. This paper proposes a realtime energy management strategy for the electric turbocharger. A multi-variable explicit model predictive controller is designed to regulate the key variables in the engine air system, while the optimal setpoints of those variables are generated by a high level controller. The controllers work in a highly efficient way to achieve the optimal energy management. This strategy has been validated in simulations and experiments. Excellent tracking performance and high robustness demonstrate the effectiveness of the proposed method.
\end{abstract}

\section{INTRODUCTION}

The growing concern of global warming due to the greenhouse gas (GHG) emissions have raised great attention in improving the energy efficiency of the transport sector. Internal combustion engines (ICE) will continue to dominate transport systems for several decades [1]. In ICE, 30-37\% fuel energy is lost in exhaust gas, while this figure can be significantly reduced by using advanced turbocharger technologies [2].

In traditional turbochargers, the turbine is driven by the exhaust gas, and the compressor connected to the turbine is rotated. As a result, the fresh air is compressed into the engine. This layout recovers part of the exhaust gas energy otherwise lost, but has two main drawbacks. First, the amount of recovered energy at a specific engine operating point is limited by the compressor power demand to deliver the desired torque. Second, the engine torque response is constricted at low engine speeds due to the turbo lag. The electric turbocharger (ET) is a promising solution to overcome these drawbacks. In the ET, an extra-high-speed electric machine (EM) is mounted on the shaft connecting the turbine and compressor wheels. This layout improves the fuel efficiency by running the EM as a generator, and improves the transient response by running the EM as a motor. The ET is a cost effective approach to improve transient response [3], [4] and downsize engines [5], [6].

The application of ETs have been generalised from road vehicles to marine vehicles [7]. Several types of EMs includ-

D. Zhao, R. Stobart and B. Mason are with Department of Aeronautical and Automotive Engineering, Loughborough University, Loughborough, Leicester, U.K. (e-mail: d.zhao@lboro.ac.uk, r.k.stobart@lboro.ac.uk, b.mason2@lboro.ac.uk).

This work was co-funded by Innovate UK, under a grant for the Low Carbon Vehicle IDP4 Programme (TP14/LCV/6///BG011L). This work was also co-funded by Engineering and Physical Sciences Research Council of U.K. under the EPSRC-UKRI Innovation Fellowship scheme (EP/S001956/1). ing the switched reluctance machine (SRM) [8], induction machine (IM) [9], and permanent magnet synchronous machine (PMSM) [10] have been used for the manufacturing of the EM within the ET. A performance overview of those EMs is given in [11]. Several milestone studies on the characterisation have also been completed. The engine fuel efficiency is impacted by the pumping losses which vary when the ET works as a motor or generator [12]. It has been shown that the ET optimal working condition can be determined by the exhaust pressure [13]. In steady state, the efficiency of the turbine and EM are both characterised [14]. The ET decouples the intake manifold and exhaust manifold, which has been verified by multiple studies [15]. Furthermore, the controllability of the ET has been proved in [16].

High efficient real-time energy management and control are the keys to the success of the ET. Meanwhile, maintaining the state-of-charge (SOC) level of the onboard battery in a reasonable range is essential. Real-time energy management and control of the ET are less developed compared to the progress in manufacturing and characterisation. This paper presents an attempt to address the gap. Recently, a consortium led by Caterpillar developed an ET for heavyduty diesel engines, named the Electric Turbocharger Assist (ETA). This study is based on an 7.01 L ETA-equipped heavy-duty diesel engine. An integrated real-time energy management strategy is designed in a hierarchical structure. At the high level, the desired setpoints of key performance variables are computed to distribute energy flows in the optimal way. At the lower level, the actuators are regulated using the explicit model predictive control (EMPC) method [17] to achieve the desired energy flow distribution in a centralized way.

\section{SySTEM DESCRIPTION}

The diagram of an electric turbocharged diesel engine (ETDE) is illustrated in Fig. 1, while the critical variables and parameters are listed in Table I. The ETA is a single stage turbocharger consisting of a variable geometry turbine (VGT), an EM and a compressor, which are mounted on the same turbine shaft. All exhaust gas passes through the ETA turbine except the exhaust gas recirculation (EGR). The expansion of the exhaust gas across the VGT rotates the compressor, and thereafter the fresh air is pumped into the intake manifold. When the EM runs in the generating mode, some of the exhaust gas energy is used to charge the battery and the turbine speed drops. This raises the exhaust pressure and exhaust gas temperature. On the other hand, when the 


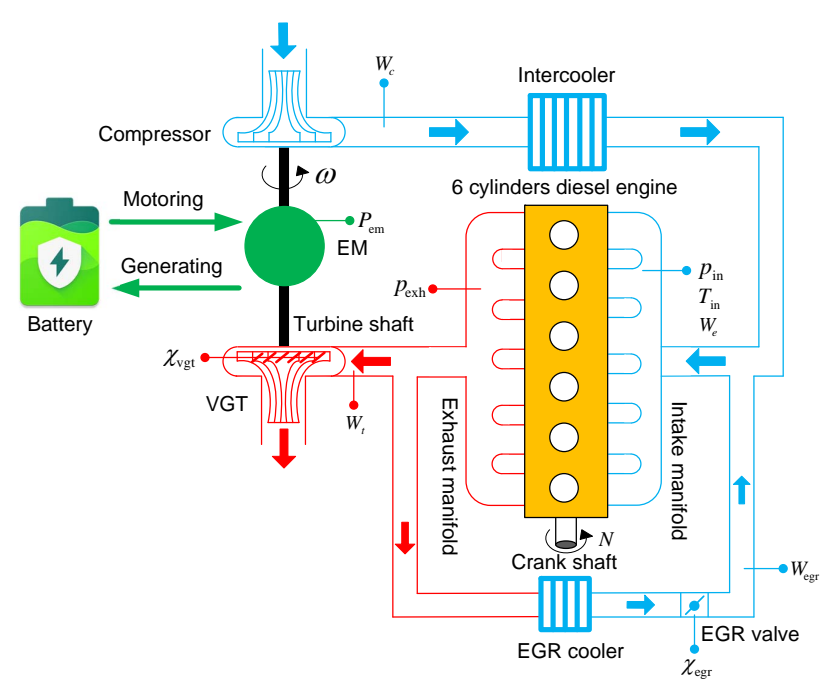

Fig. 1. Electric turbocharged diesel engine.

EM runs in the motoring mode, both of the exhaust pressure and exhaust gas temperature drops. The VGT moves toward close to ensure sufficient energy is used for air compression. A SRM is used as the EM because of its simple structure and the resulting low cost. The SRM has a rated power of 5 $\mathrm{kW}$.

According to the conservation laws of mass and energy, the engine air system dynamics can be determined by the pressure and mass changes in the intake and exhaust manifolds: $\dot{p}_{\text {in }}, \dot{p}_{\text {exh }}, \dot{m}_{\text {in }}$ and $\dot{m}_{\text {exh }}$ [18]. Strong nonlinearities exist in the air system for the mass flow exchanges among the EGR, VGT, compressor, and manifolds. The mass flow rates can be expressed as:

$$
\begin{array}{ll}
W_{\text {in }}=W_{e}+W_{f}, & W_{\text {exh }}=W_{\text {egr }}+W_{t} \\
W_{e}=f\left(p_{\text {in }}, T_{\text {in }}, \eta_{v}, N\right), & W_{\text {egr }}=f\left(p_{\text {in }}, p_{\text {exh }}, \chi_{\text {egr }}\right) \\
W_{t}=f\left(p_{\text {exh }}, T_{\text {exh }}, \chi_{\text {vgt }}\right), & W_{c}=f\left(p_{\text {in }}, \omega\right) .
\end{array}
$$

$W_{e}$ is modeled using the speed-density equation; $W_{\text {egr }}$ is modeled as the standard orifice equation; $W_{t}$ and $W_{c}$ are modeled as nonlinear functions with respect to the efficiencies $\eta_{t}$ and $\eta_{c}$. The dynamics of the turbocharger is represented by the mechanical equation:

$$
\dot{\omega}=\left(\eta_{m}\left(P_{t}+P_{\mathrm{em}}\right)-P_{c}\right) /(J \omega)
$$

where $\eta_{m}$ quantifies the bearing power losses on the turbine shaft and windage losses in the EM. The power of both compressor and turbine are represented by maps with respect to $\eta_{t}$ and $\eta_{c}$ :

$$
\begin{gathered}
P_{t}=f\left(W_{t}, T_{\text {exh }}, p_{\text {exh }}, \eta_{t}\right), \quad P_{c}=f\left(W_{c}, p_{\text {in }}, p_{a}, \eta_{c}\right) . \\
\text { III. ReAL-Time ENERGy ManaGement } \\
\text { STRATEGy DeSign }
\end{gathered}
$$

Real-time energy management is to distribute the energy flows in an optimal way. It is achieved by online generating and tracking the optimal setpoints of $p_{\text {in }}, p_{\text {exh }}$ and $W_{\text {egr }}$, denoted as $p_{\text {in }}^{\star}, p_{\text {exh }}^{\star}$ and $W_{\text {egr }}^{\star}$. According to the vehicle exhaust
TABLE I

NOMENCLATURE

\begin{tabular}{lll}
\hline Variable & Description & $\mathrm{Unit}$ \\
\hline$N$ & Engine speed & $\mathrm{r} / \mathrm{min}$ \\
$\omega$ & Turbine speed & $\mathrm{rad} / \mathrm{s}$ \\
$T_{L}$ & Engine load & $\mathrm{N} \cdot \mathrm{m}$ \\
$W_{f}$ & Engine fuelling rate & $\mathrm{kg} / \mathrm{s}$ \\
$W_{e}$ & Total mass flow rate to cylinders & $\mathrm{kg} / \mathrm{s}$ \\
$W_{c}$ & Compressor air flow rate & $\mathrm{kg} / \mathrm{s}$ \\
$W_{\text {egr }}$ & EGR mass flow rate & $\mathrm{kg} / \mathrm{s}$ \\
$W_{t}$ & Gas gas mass flow rate through the turbine & $\mathrm{kg} / \mathrm{s}$ \\
$P_{c}$ & Compressor power & $\mathrm{kW}$ \\
$P_{t}$ & Turbine power & $\mathrm{kW}$ \\
$P_{b}$ & Engine brake power & $\mathrm{kW}$ \\
$P_{\text {em }}$ & EM power & $\mathrm{kW}$ \\
$T_{\text {in }}$ & Intake manifold temperature & $\mathrm{K}$ \\
$T_{\text {exh }}$ & Exhaust manifold temperature & $\mathrm{K}$ \\
$T_{\text {egr }}$ & Mass flow temperature at the EGR outlet & $\mathrm{K}$ \\
$T_{c}$ & Air temperature at the compressor outlet & $\mathrm{K}$ \\
$p_{\text {in }}$ & Boost pressure & $\mathrm{kPa}$ \\
$p_{\text {exh }}$ & Exhaust manifold pressure & $\mathrm{kPa}$ \\
$p_{a}$ & Ambient pressure & $\mathrm{kPa}$ \\
$m_{\text {in }}$ & Mass in the intake manifold & $\mathrm{kg}$ \\
$m_{\text {exh }}$ & Mass in the exhaust manifold & $\mathrm{kg}$ \\
$V_{\text {in }}$ & Intake manifold volume & $\mathrm{m}$ \\
$V_{\text {exh }}$ & Exhaust manifold volume & $\mathrm{m} 3$ \\
$J$ & Turbine shaft moment of inertia & $\mathrm{kg} \cdot \mathrm{m}^{2}$ \\
$\eta_{c}$ & Compressor isentropic efficiency & - \\
$\eta_{t}$ & Turbine isentropic efficiency & - \\
$\eta_{v}$ & Volumetric efficiency & - \\
$\eta_{m}$ & Turbocharger mechanical efficiency & - \\
$\chi_{\text {egr }}$ & EGR valve opening & - \\
$\chi_{\mathrm{vgt}}$ & VGT vane opening & - \\
\hline & &
\end{tabular}

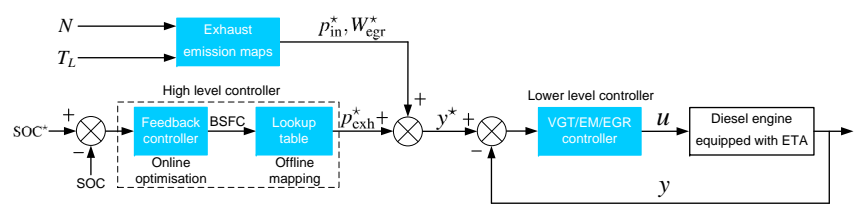

Fig. 2. Engine control and energy management structure.

emission standards on $\mathrm{NO}_{x}$ and particulate matter (PM), $p_{\text {in }}^{\star}$ and $W_{\text {egr }}^{\star}$ are generated from offline characterisation. To maintain the battery SOC within a special range while minimising the brake specific fuel consumption (BSFC), $p_{\mathrm{exh}}^{\star}$ would be updated online. The proposed strategy consists of high and lower level controllers as shown in Fig. 2, where $\mathrm{SOC}^{\star}$ is the desired value of SOC. The high level controller generates the optimal setpoints which would be tracked by employing the lower level controller.

\section{A. Lower Level Controller Design}

The EMPC is selected as the lower level controller for its capability in dealing with internal couplings and external constraints. The controller is synthesized offline using the multi-parametric quadratic programming (mpQP) method based on a linear model. The model is identified from calibration data. Afterwards, the synthesized controller will be run on the engine control unit (ECU). In the online implementation, the optimal control laws will be invoked according to engine feedback signals. The complete procedure in compiling and implementing the EMPC is illustrated in Fig. 3. 


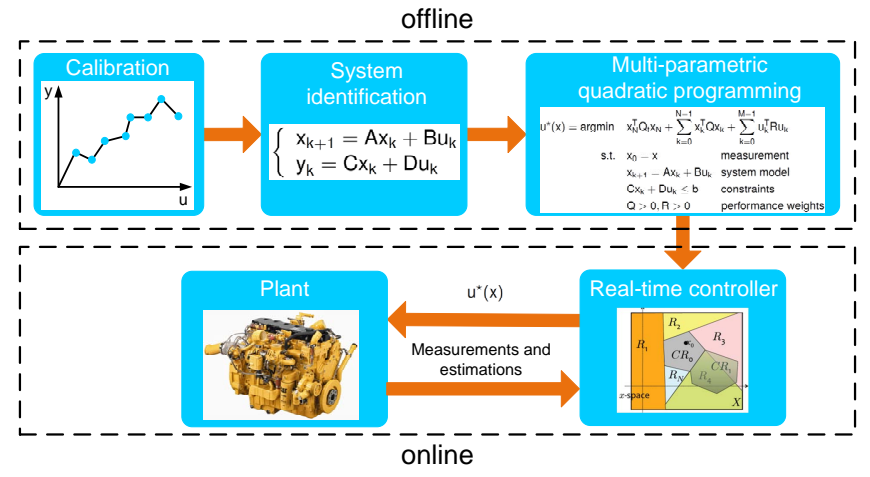

Fig. 3. Explicit model predictive control solution.

1) Problem Formulation: The ETDE model is highly nonlinear due to the couplings among multiple loops. By segregating the entire engine operating region into multiple local zones, the ETDE model in one local zone can be linearised as

$$
\left\{\begin{array}{l}
x(k+1)=A x(k)+B u(k)+B_{d} d(k) \\
y(k)=C x(k)+D_{d} d(k)
\end{array}\right.
$$

where $x \in \mathbb{R}^{n}, u \in \mathbb{R}^{3}$, and $y \in \mathbb{R}^{3}$ are the state vector, input vector, and output vector, respectively; $d$ is the disturbance; $A, B$, and $C$ are coefficient matrices to be identified. The inputs and outputs are selected as

$$
u=\left[\begin{array}{lll}
\chi_{\mathrm{vgt}} & P_{\mathrm{em}} & \chi_{\mathrm{egr}}
\end{array}\right]^{\mathrm{T}}, \quad y=\left[\begin{array}{lll}
p_{\mathrm{in}} & p_{\mathrm{exh}} & W_{\mathrm{egr}}
\end{array}\right]^{\mathrm{T}} .
$$

The control problem is defined as the following optimisation problem:

$$
\begin{aligned}
U^{\star}(k)=\operatorname{argmin} & \sum_{i=0}^{H_{p}}\left\|y(k+i)-y^{\star}(k)\right\|_{Q}^{2}+\|\Delta u(k+i)\|_{R}^{2} \\
\text { s.t. } & x(k+1)=A x(k)+B u(k)+B_{d} d(k) \\
& u(k)=u(k-1)+\Delta u(k) \\
& y(k)=C x(k)+D_{d} d(k) \\
& u_{\min } \leq u \leq u_{\max } \\
& y_{\min } \leq y \leq y_{\max }
\end{aligned}
$$

where

$$
\begin{aligned}
U(k) & \triangleq\left[\Delta u^{\mathrm{T}}(k), \ldots, \Delta u^{\mathrm{T}}\left(k+H_{c}-1\right)\right]^{\mathrm{T}} \\
\Delta u(k+i) & =\Delta u\left(k+H_{c}-1\right), \quad i=H_{c}, \ldots, H_{p} \\
Q & =Q^{\mathrm{T}}>0 \\
R & =R^{\mathrm{T}}>0 \\
\|x\|_{Q}^{2} & =x^{\mathrm{T}} Q x .
\end{aligned}
$$

The constraints on $u$ are the amplitude boundaries of the control signals, and the constraints on $y$ represent the physical ranges of the outputs, where $u$ and $y$ have been defined in (3). $H_{p}$ and $H_{c}$ are the prediction horizon and control horizon respectively and $H_{p} \geq H_{c}$ is held.

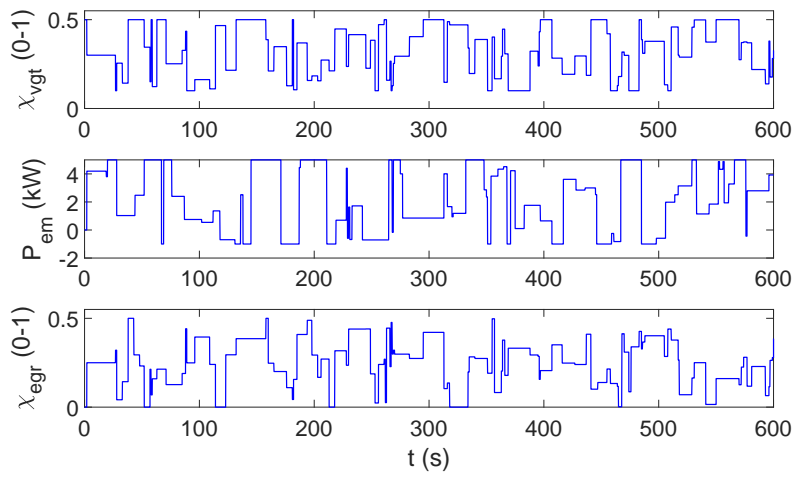

(a)
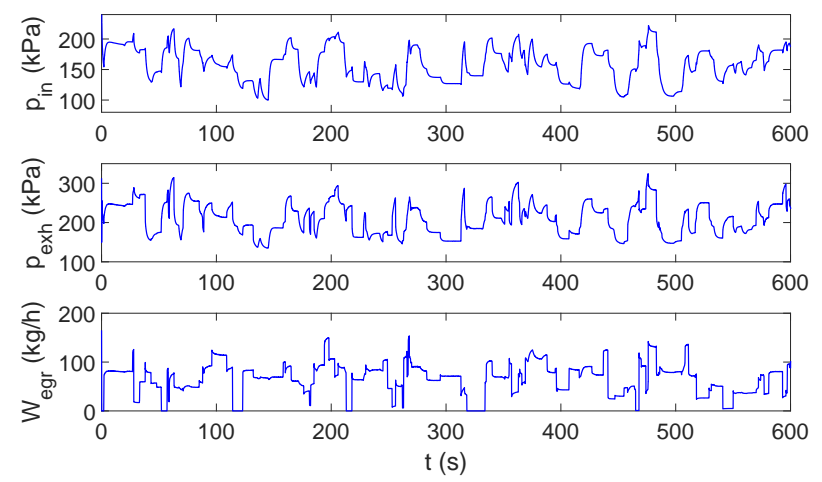

(b)
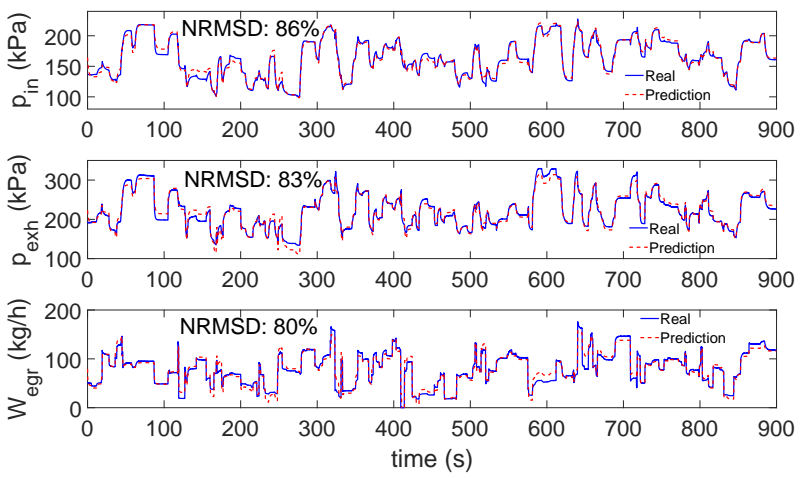

(c)

Fig. 4. Training and validation data at $(1800 \mathrm{r} / \mathrm{min}, 120 \mathrm{~N} \cdot \mathrm{m})$ for model identification. (a) Training inputs. (b) Training outputs. (c) Validation outputs.

2) Model Identification: Two independent data sets are generated for model identification. As an example, the experimental training and validation data at $1800 \mathrm{r} / \mathrm{min}, 120 \mathrm{~N} \cdot \mathrm{m}$ are illustrated in Fig. 4. In the training set, the constraints on inputs are

$u_{\min }=\left[\begin{array}{lll}0.1 & -1.5 \mathrm{~kW} & 0\end{array}\right]^{\mathrm{T}}, u_{\max }=\left[\begin{array}{lll}0.5 & 4.5 \mathrm{~kW} & 0.5\end{array}\right]^{\mathrm{T}}$

as shown in Fig. 4(a). The corresponding engine outputs are plotted in Fig. 4(b). The ssest solver in MATLAB is used to identify the linear model. The normalised root-mean-square deviation (NRMSD) is used to evaluate the identified model. 
For the $i$-th variable in $y$, the NRMSD is

$$
J=1-\sqrt{\sum_{i=1}^{m}\left(y_{v}(i)-y_{p}(i)\right)^{2}} / \sqrt{\sum_{i=1}^{m}\left(y_{v}(i)-\bar{y}_{v}\right)^{2}}
$$

where $y_{v}$ is the validation data, $y_{p}$ is the model output, $\bar{y}_{v}$ is the mean value of $y_{v}$, and $m$ is the number of data samples. The model order should not be too high to prevent overfitting. In this case, a 3rd-order model is identified while the NRMSD on $p_{\text {in }}, p_{\text {exh }}$ and $W_{\text {egr }}$ are $86 \%, 83 \%$ and $80 \%$. Model validation results are given in Fig. 4(c).

3) Controller Synthesis: Online optimisation in standard MPC is computationally intensive. This issue can be addressed by solving (4) offline over predefined ranges. Substituting (2) into the cost function of (4), the optimisation problem can be re-formulated as

$$
\begin{aligned}
U^{\star}(k)=\operatorname{argmin} & \frac{1}{2} U(k)^{\mathrm{T}} H U(k)+x(k)^{\mathrm{T}} F U(k) \\
\text { s.t. } & G U(k) \leq W+E x(k)
\end{aligned}
$$

where $H, F, G, W, E$ are algebraically transformed from $Q, R$ and system model (2). Introducing $z(k) \triangleq U(k)+$ $H^{-1} F^{\mathrm{T}} x(k)$, then (7) is transformed into the following form:

$$
\begin{aligned}
z^{\star}(k)=\operatorname{argmin} & \frac{1}{2} z(k)^{\mathrm{T}} H z(k) \\
\text { s.t. } & G z(k) \leq W+S x(k)
\end{aligned}
$$

where $S \triangleq E+G H^{-1} F^{\mathrm{T}}$. The solution $z^{\star}(k)$ is a continuous piecewise affine (PWA) function defined over a polyhedral partition, so is the $U^{\star}(k)$ :

$$
U^{\star}(k)= \begin{cases}f_{1} x(k)+g_{1} & \text { if } h_{1} x(k) \leq k_{1} \\ & \vdots \\ f_{m} x(k)+g_{m} & \text { if } h_{m} x(k) \leq k_{m}\end{cases}
$$

where the inequalities mean the constraints on the polygons, and $m$ is the number of polygons. The MATLAB Model Predictive Control Toolbox is used to generate EMPC control laws. The control laws are stored in look-up tables within the ECU. In online implementation, control laws are read from the table without conducting any optimisation. This grants the EMPC the advantage of reduced computation time meanwhile maintaining identical performance with the MPC. In real-time control, only the first component of $U^{\star}(k)$ would be applied:

$$
u(k)=\left[\begin{array}{llll}
I & 0 & \ldots & 0
\end{array}\right] U^{\star}(k) .
$$

In the next sampling step, $U^{\star}(k+1)$ is called from the lookup table again, and $u(k+1)$ is updated accordingly.

\section{B. High Level Controller Design}

The high level controller generates $p_{\text {exh }}^{\star}$ according to offline mapping and online optimisation. In the offline mapping, the relationships among $p_{\mathrm{exh}}^{\star}, P_{\mathrm{em}}$ and BSFC are found. In the online optimisation, a feedback controller regulates the battery SOC while maximising the energy efficiency.

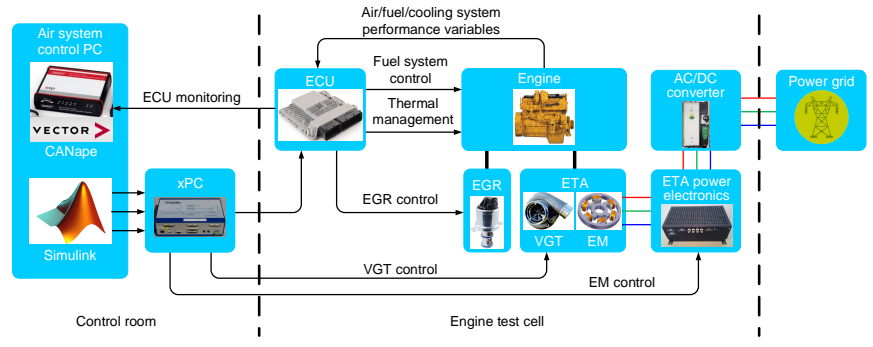

Fig. 5. Experimental setup.

1) Problem Formulation: In the ETA, the following power balance equation holds:

$$
P_{c}=P_{\mathrm{em}}+P_{t} .
$$

To reveal the impact of battery SOC deviation on engine running, an equivalent power is defined as

$$
P_{\mathrm{eq}}=s(\mathrm{SOC}) P_{\mathrm{em}}+P_{t} .
$$

Traditionally, the engine fuel economy is quantified by BSFC: $\Theta=W_{f} / P_{b}$. In the ETDE, an equivalent BSFC is defined as $\Theta_{e}=\Theta P_{\mathrm{eq}} / P_{t}$. The control problem is formulated as

$$
\begin{aligned}
p_{\text {exh }}^{\star}=\operatorname{argmin} & \Theta_{e} \\
\text { s.t. } & u_{\min } \leq u \leq u_{\max } .
\end{aligned}
$$

2) Offline Mapping: The offline mapping is completed at specific engine operating points. In this process, $p_{\text {exh }}^{\star}$ is increased in a staircase manner, while $p_{\text {in }}^{\star}$ and $W_{\text {egr }}^{\star}$ are generated by the ECU. The developed EMPC controller is used to track the updated $y^{\star}$. The values of $p_{\text {exh }}^{\star}$ will be feasible only if $p_{\text {exh }}^{\star}, p_{\text {in }}^{\star}$ and $W_{\text {egr }}^{\star}$ can all be tracked for $u \in\left[u_{\min }, u_{\max }\right]$. For each feasible $p_{\text {exh }}^{\star}$, the values of $\Theta, P_{t}$ and $P_{\mathrm{em}}$ will be recorded.

3) Online Optimisation: $p_{\mathrm{exh}}^{\star}$ varies according to different $s(\mathrm{SOC})$ values which impact $\Theta_{e}$. To recover the battery SOC in real-time, the equivalent factor in (12) is designed as

$$
s(\mathrm{SOC})=K \Delta \mathrm{SOC}
$$

where $K$ is a positive constant to be designed, $\triangle \mathrm{SOC}=$ $\mathrm{SOC}-\mathrm{SOC}^{\star}$, and $\mathrm{SOC}^{\star}$ is the desired value of SOC. At different SOC values, $P_{t}$ and $P_{\mathrm{em}}$ have different weights on $\Theta_{e}$, and then $p_{\mathrm{exh}}^{\star}$ is read from the lookup table that corresponds to $\Theta_{e}^{\star}$ which holds the minimal value.

\section{VALidation Results}

The experimental setup is based on a Cat C7.1 ACERT heavy-duty off-highway diesel engine as shown in Fig. 5. The proposed controller is built in Simulink, where EGR control signal is sent via the ECU, and other control signals are sent from a target XPC. The calibration results from experiments have been used to set up the high fidelity physical engine model in Dynasty. Dynasty is a proprietary multi-physics simulation software developed by Caterpillar, in which the engine manifolds are modeled as 1-D components. The lower level controller was evaluated in Dynasty, while the 

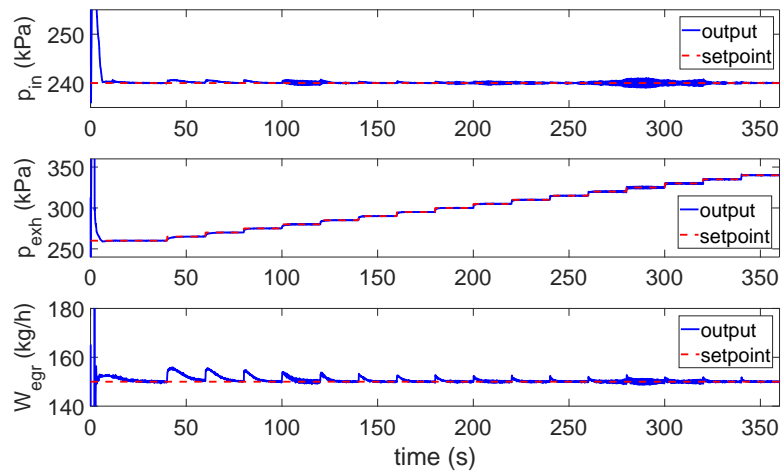

(a)
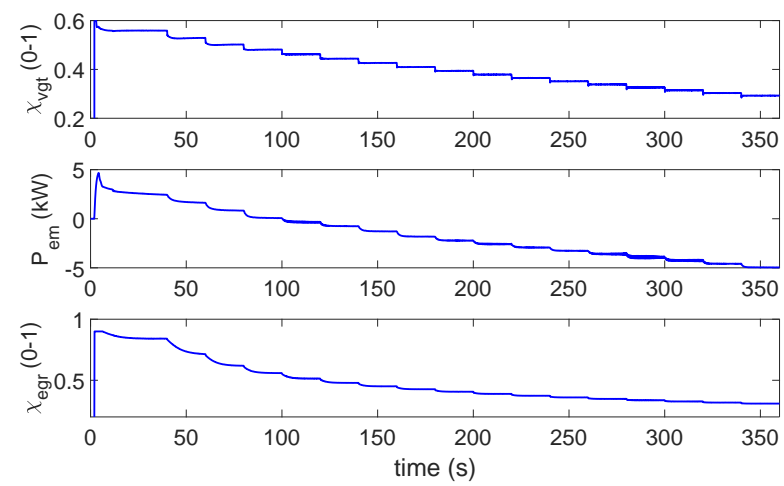

(b)

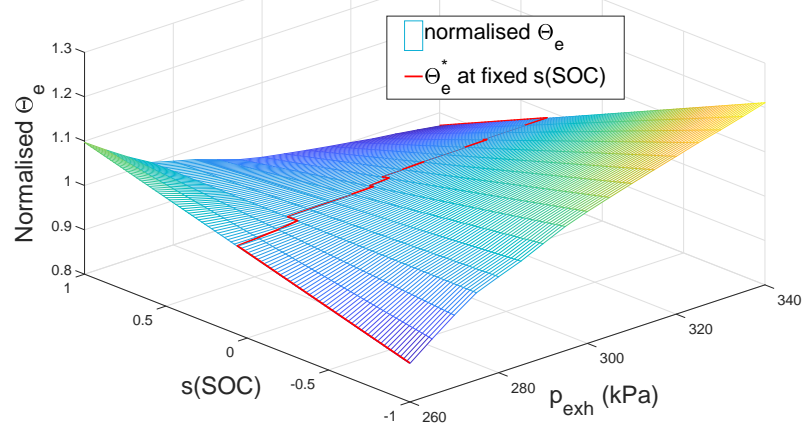

(c)

Fig. 6. Control results at $(1800 \mathrm{r} / \mathrm{min}, 800 \mathrm{~N} \cdot \mathrm{m})$ and the online adaptation of $p_{\text {exh }}^{\star}$ with variable $s(\mathrm{SOC})$. (a) Outputs regulation. (b) Control signals. (c) The trajectory of $\Theta^{\star}$ with respect to $s(\mathrm{SOC})$ and $p_{\text {exh }}$.

energy management strategy and controller robustness were evaluated in experimental testing.

\section{A. Evaluation of the Lower Level Controller}

The control performance at $(1800 \mathrm{r} / \mathrm{min}, 800 \mathrm{~N} \cdot \mathrm{m})$ are illustrated in Fig. 6. The control signals are constrained by $u_{\min }=\left[\begin{array}{lll}0.1 & -5 \mathrm{~kW} & 0\end{array}\right]^{\mathrm{T}}$ and $u_{\max }=\left[\begin{array}{lll}0.7 & 5 \mathrm{~kW} & 1\end{array}\right]^{\mathrm{T}}$. All the command signals are tracked well when $p_{\mathrm{exh}}^{\star} \in$ [260 kPa, $340 \mathrm{kPa}]$ as in Fig. 6, while $p_{\text {in }}^{\star}$ and $W_{\text {egr }}^{\star}$ are 240 $\mathrm{kPa}$ and $150 \mathrm{~kg} / \mathrm{h}$ respectively. $P_{\mathrm{em}}$ will be less than $-5 \mathrm{~kW}$ if $p_{\mathrm{exh}}^{\star}>340 \mathrm{kPa}$, which is out of the permissible range of $u$. From Fig. 6(b), it can be observed that the ETA has more margin to recover energy at high load. The $p_{\text {exh }}^{\star}$ values at

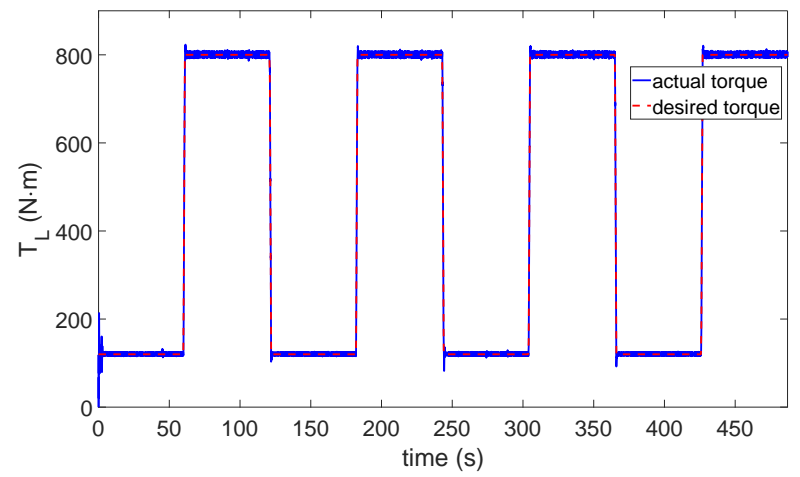

(a)

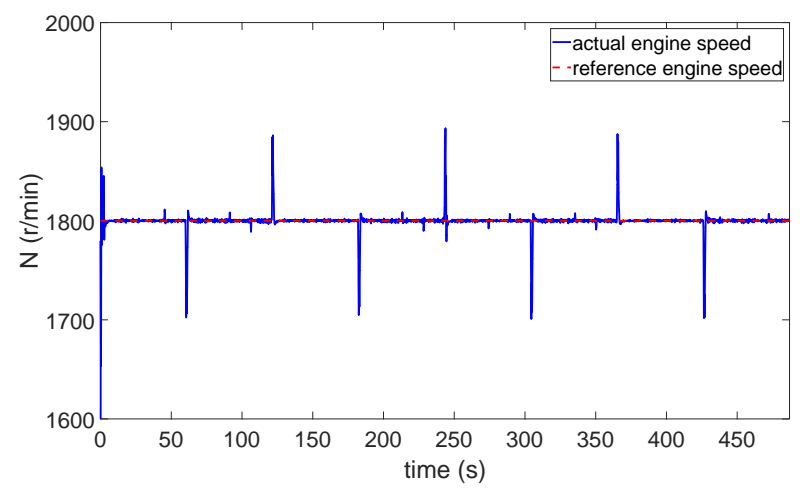

(b)

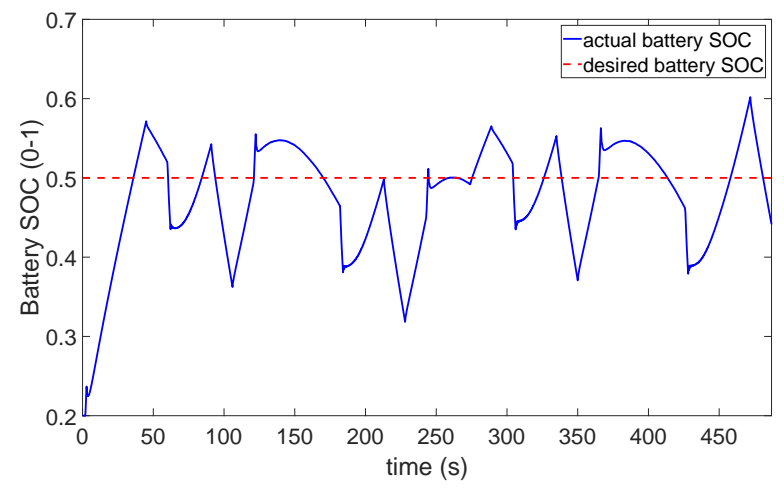

(c)

Fig. 7. Evaluation of the energy management strategy. (c) $T_{L}$. (b) $N$. (c) SOC.

different $s$ are ploted in Fig. 6(c).

\section{B. Evaluation of the Energy Management Strategy}

The integrated energy management strategy is evaluated under block loads between $120 \mathrm{~N} \cdot \mathrm{m}$ and $800 \mathrm{~N} \cdot \mathrm{m}$, while the load profile is shown in Fig. 7(a). The maximum EM motoring power is applied in load acceptance, and the maximum EM motoring power is applied in load shedding. This setting refrains the oscillation on $N$ less than $100 \mathrm{r} / \mathrm{min}$, as shown in Fig. 7(b). The SOC is regulated to vary around the desired value as shown in Fig. 7(c), while $\mathrm{SOC}^{\star}=0.5$ and the SOC initial value is 0.2 . This is achieved by updating and tracking of the $p_{\mathrm{exh}}^{\star}$ in real-time. 


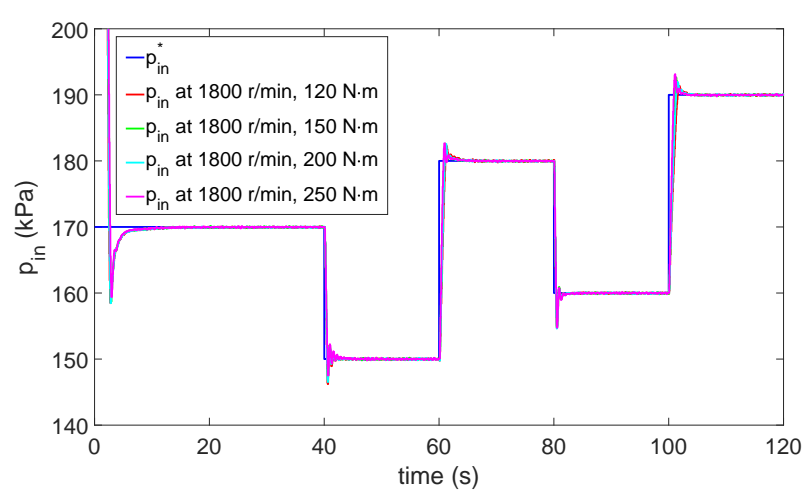

(a)

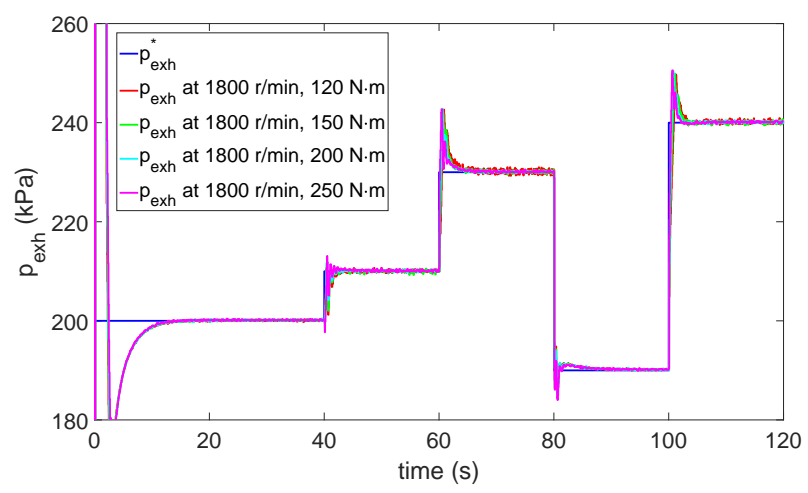

(b)

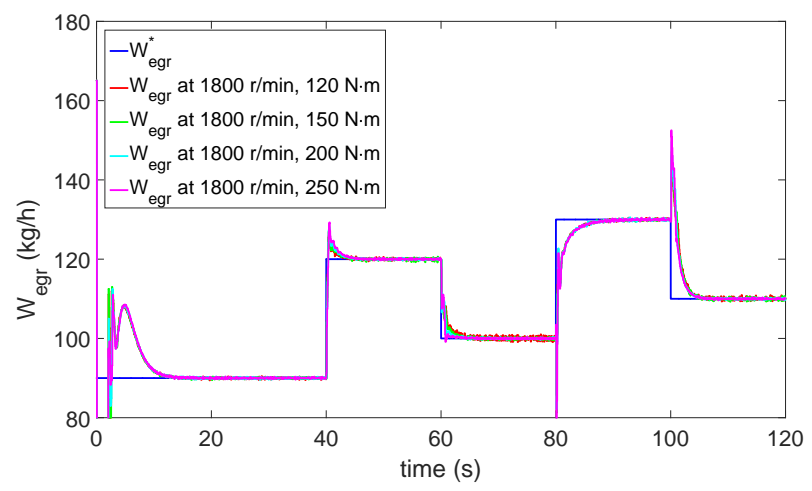

(c)

Fig. 8. Evaluation of the controller robustness at different operating points. (a) $p_{\text {in }}$. (b) $p_{\text {exh }}$. (c) $W_{\text {egr }}$. (d) $\chi_{\text {vgt }}$. (e) $P_{\text {em }}$. (f) $\chi_{\text {egr }}$.

\section{Evaluation under Multiple Operating Points}

The EMPC controller should hold robustness because it is required to be both effective at a single operating point and over a region. In Fig. 8, the controller was tested under four different operating points, while the torque ranges from $120 \mathrm{~N} \cdot \mathrm{m}$ to $250 \mathrm{~N} \cdot \mathrm{m}$. At all of the four operating points, the setpoints are tracked very well, as shown in Fig. 8(a) - Fig. 8(c). This means that the EMPC controller is effective in a local zone once the actuators are not saturated.

\section{CONCLUSION}

A real-time energy management strategy on an electric turbocharger for heavy-duty ground vehicles is proposed in this paper. An EMPC controller is analytically designed to track the setpoints of selected outputs in a systematic way. A high level controller is designed to update the exhaust pressure setpoints online. The ETDE energy efficiency is consequently maximised while the battery SOC is maintained around the desired value. Promising validation results are provided in physical simulations and experiments.

\section{REFERENCES}

[1] G. Kalghatgi, "Is it really the end of internal combustion engines and petroleum in transport?" Appl. Energy, vol. 225, pp. 965-974, 2018.

[2] S. Chu et al., "Opportunities and challenges for a sustainable energy future," Nature, vol. 488, pp. 294-303, 2012.

[3] S. Ebbesen, "Time-optimal control strategies for a hybrid electric race car," IEEE Trans. Control Syst. Technol., vol. 26, pp. 233-247, 2018.

[4] Y. Yashiro et al., "High speed model predictive control for nextgeneration turbocharging system," Mitsubishi Heavy Industries Technical Review, vol. 54, no. 1, pp. 77-81, 2017.

[5] X. Xue et al., "Potentials of electrical assist and variable geometry turbocharging system for heavy-duty diesel engine downsizing," $S A E$ International, pp. 2017-01-1035, 2017.

[6] A. Mamat et al., "Characterisation of a low pressure turbine for turbocompounding applications in a heavily downsized mild-hybrid gasoline engine," Energy, pp. 3-16, 2014.

[7] D. Singh, "A review of waste heat recovery technologies for maritime applications," Energy Convers. Manage., vol. 111, pp. 315-328, 2016.

[8] B. Hanko et al., "The use of high speed switched reluctance machine for electric turbochargers," in Proceedings of the 25th International Workshop on Electric Drives, 2017, pp. 207-212.

[9] D. Gerada et al., "Design aspects of high speed high power density laminated rotor induction machines," IEEE Trans. Ind. Electron., vol. 58, no. 9, pp. 4039-4047, 2011.

[10] F. Crescimbini, "High-speed electric drive for exhaust gas energy recovery applications," IEEE Trans. Ind. Electron., vol. 61, no. 6, pp. 2998-3011, 2014.

[11] W. Lee et al., "Overview of electric turbocharger and supercharger for downsized internal combustion engines," IEEE Trans. Mechatronics, vol. 22, no. 2, pp. 774-784, 2017.

[12] E. Winward et al., "Performance testing of an electrically assisted turbocharger on a heavy duty diesel engine," in Proc. 12th Int. Conf. Turbochargers Turbocharging, 2016.

[13] R. Kiwan et al., "Thermodynamic and practical benefits of waste energy recovery using an electric turbo-generator under different boosting methods," SAE Int., pp. 2018-01-0851, April 2018.

[14] N. Terdich et al., "Experimental efficiency characterization of an electrically assisted turbocharger," SAE Int., pp. 2013-24-0122, August 2013.

[15] H. Aghaali et al., "A review of turbocompounding as a waste heat recovery system for internal combustion engines," Renew. Sustain. Energy Rev., vol. 49, pp. 813-824, 2015.

[16] D. Zhao et al., "An integrated framework on characterization, control and testing of an electrical turbocharger assist," IEEE Trans. Ind. Electron., vol. 65, no. 6, pp. 4897-4908, 2018.

[17] A. Bemporad, M. Morari, V. Dua, and E. Pistikopoulos, "The explicit linear quadratic regulator for constrained systems," Automatica, vol. 38, pp. 3-20, 2002.

[18] B. Glenn et al., "Control design of electrically assisted boosting systems for diesel powertrain applications," IEEE Trans. Control Syst. Technol., vol. 18, no. 4, pp. 769-778, 2010. 